\title{
Las preferencias de interacción social en las Escuelas Sociodeportivas de Baloncesto de la Fundación Real Madrid The preferences of social interaction in the Basketball Socio-sport Schools of the Real Madrid Foundation
}

\author{
*Gema Ortega Vila, **José Robles Rodríguez, **Manuel Tomás Abad Robles, ***Luis Javier Durán González, *Jorge Franco \\ Martín, ***Ana Concepción Jiménez Sánchez, ** Fco. Javier Giménez Fuentes-Guerra \\ *Fundación Real Madrid (España), **Universidad de Huelva (España), *** Universidad Politécnica de Madrid (España)
}

\begin{abstract}
Resumen. El objetivo del presente estudio fue analizar las preferencias de interacción social de los chicos y chicas de las Escuelas Sociodeportivas de Baloncesto de la Fundación Real Madrid. La muestra estaba formada por 413 jugadores, 68\% (n=281) chicos y 32\% (n=132) chicas. La distribución por categorías fue, el 31,2\% pertenecían a la categoría Benjamín (n=129), el 36,1\% a la categoría alevín (n=149), el 25,2\% a la categoría infantil ( $n=104)$ y, el 7,5\% a la categoría cadete $(n=31)$. El instrumento utilizado fue la Escala Graupera/Ruiz de Preferencias de Interacción Social (GRSIPPEL), que explora cuatro dimensiones de preferencia de aprendizaje: Cooperación, competencia, afiliación e individualismo. Para el análisis estadístico se utilizaron pruebas no paramétricas (U de Mann-Whitney y Kruskal-Wallis), se calculó el tamaño del efecto y, para determinar si existían asociaciones entre las distintas dimensiones se utilizó el coeficiente de correlación de Spearman. Los resultados mostraron que el Proyecto DeportivoEducativo de la Fundación Real Madrid contribuye a que no existan diferencias acentuadas en función del sexo. Además, los chicos y chicas presentaban una mayor inclinación hacia las actitudes cooperativas frente a las competitivas. Por otro lado, se detectaron diferencias entre las categorías de minibasket (benjamín y alevín) y las categorías de baloncesto (infantil y cadete).
\end{abstract}

Palabras clave: Iniciación deportiva, cooperación, competición, afiliación, individualismo, baloncesto.

\begin{abstract}
The aim of this study was to analyze the social interaction preferences of boys and girls at the Real Madrid Foundation's Socio-Sports Basketball Schools. The sample consisted of 413 players, $68 \%(n=281)$ boys and $32 \%(n=132)$ girls. The distribution by category was 31 . $2 \%$ in the Benjamin category $(n=129), 36.1 \%$ in the alevin category ( $n=149), 25.2 \%$ in the infants' category $(n=104)$ and $7.5 \%$ in the youths' category $(n=31)$. The instrument used was the Graupera/Ruiz Scale of Social Interaction Preferences (GR-SIPPEL), which explores four dimensions of learning preference: Cooperation, competence, affiliation and individualism. Non-parametric tests (Mann-Whitney U-test and Kruskal-Wallis) were used for statistical analysis, the size of the effect was calculated, and Spearman's correlation coefficient was used to determine if there were associations between the different dimensions. The results showed that the Real Madrid Foundation's Sports-Educational Project contributes to the fact that there are no marked differences according to gender. In addition, boys and girls were more inclined towards cooperative attitudes than competitive ones. On the other hand, differences were detected between the categories of minibasket and the categories of basketball.
\end{abstract}

Keywords: Sports initiation, cooperation, competition, affiliation, individualism, basketball.

\section{Introducción}

La práctica deportiva en la edad escolar está relacionada con multitud de beneficios cognitivos, emocionales y sociales, que van más allá de la adquisición de unas habilidades específicas. En este sentido, muchos autores (Giménez, Abad \& Robles, 2010; Monjas, Ponce \& Gea, 2015) promueven la práctica deportiva en edades de formación como un medio que contribuye a la educación integral de los jóvenes deportistas, y no solo como un paso para el alto rendimiento. Para ello, es necesario huir de los tradicionales modelos deportivos orientados hacia el ego, y buscar alternativas en modelos orientados hacia la tarea (Granero-Gallegos \& Baena-Extremera, 2014) y, de esta forma, centrarse en las interesantes características educativas que puede tener el deporte de base cuando está bien planificado (Contreras, De la Torre \& Velázquez, 2001; Gutiérrez, 2003). En la actualidad están apareciendo nuevos modelos deportivos desde el punto de vista educativo que contribuyen al fomento de valores (Veroz, Yague \& Tabernero, 2015).

Avanzando en estas consideraciones iniciales, el modelo de Escuelas Sociodeportivas de la Fundación Real Madrid (FRM) se ha caracterizado, desde sus inicios, por unos planteamientos pedagógicos que resumimos a continuación (Ortega et al., 2012; Ortega et al., 2014): los valores educativos son el eje central sobre el que se sustenta el modelo; inclusión y no selección ni discriminación de ningún tipo en los participantes (nivel, género o discapacidad); perfil educativo de los entrenadores y entrenadoras; competición adaptada (reglas, instalación, materiales, $\mathrm{n}^{\mathrm{o}}$ participantes) donde prima la participación y no el

Fecha recepción: 24-01-18. Fecha de aceptación: 25-06-18 Javier Giménez Fuentes-Guerra jfuentes@uhu.es resultado; e implicación de las familias en la evolución del modelo. Además, desde la propia Fundación, se realizan diferentes actuaciones formativas dirigidas al profesorado, a las familias y al alumnado participante en dichas escuelas deportivas. Para valorar la repercusión social y educativa, cada uno de los programas que componen el proyecto está siendo analizado a través de diferentes instrumentos, entre los que destaca el elaborado por Ruiz, Graupera, Moreno y Rico (2010), el cual pretende diagnosticar las preferencias de interacción social, haciendo hincapié en el estudio de las actitudes cooperativas, competitivas, individualistas y afiliativas en el ámbito educativo, siendo esta escala la que hemos utilizado en el trabajo que aquí se presenta.

Respecto al fomento de la cooperación, André, Deneuve y Louvet (2011) detectaron que el aprendizaje cooperativo influyó positivamente en la aceptación de los estudiantes con discapacidad por parte del resto de los alumnos en las clases de educación física. Oortwijn, Boekaert, Vedder y Fortuin (2008) señalaron que el aprendizaje a través de las formas cooperativas es un medio que favorece la integración entre chicos/as de diferentes culturas, contribuyendo a amistades interétnicas más fructíferas. En el ámbito deportivo aparecen estudios relacionados con el fomento de la cooperación en busca de mejoras en las habilidades deportivas (Dyson, 2000). Fomentar la cooperación supone trabajar en grupos heterogéneos en los que los jugadores aúnan esfuerzos y comparten recursos para mejorar su propio aprendizaje y también el de los demás miembros del equipo (Velázquez, 2015).

Por otra parte, la competición durante la etapa de formación deportiva contribuye, en ocasiones, a que los niños/as presenten situaciones de estrés derivadas de la misma (Prieto, 2016). De Rose Jr., Korsakas, Carlstron y Ramos, (2000), en su estudio con 723 jóvenes brasileños entre 10 y 17 años de edad, observaron que los chicos/as que participaban en eventos de- 
portivos a nivel competitivo mostraron niveles más altos de síntomas de estrés, siendo superiores en el sexo femenino. Prat y Soler (2003) consideran que la competitividad conlleva muchas contradicciones ya que favorece, en función de cómo se lleve a cabo, actitudes positivas y negativas en el proceso educativo del sujeto. Por ello, es importante estudiar y orientar las conductas de los jóvenes. En la misma línea, según Harvey, Kirk, y O’Donovan (2014), el deporte en sí no va a proporcionar al joven unos valores éticos mediante el mero hecho de participar. Éstos valores deben ser planificados, ya que, adecuadamente conducido, el deporte puede otorgar a los jóvenes serias experiencias éticas (Ortega et al., 2016; Twietmeyer, 2007).

Respecto a las preferencias en cuanto al aprendizaje individualista, hay que señalar que, en principio, están plenamente enfrentadas a los planteamientos cooperativos anteriormente citados. En este sentido, García y García (2006), en su estudio con niños y niñas entre 6 y 8 años, observaron que aquéllos que presentaban mayores niveles en cuanto a sus habilidades deportivas, eran más competitivos, mostrando actitudes más individualistas y egocéntricas que el resto de compañeros.

Por último, la afiliación, la cual hace referencia al desarrollo de relaciones sociales íntimas y a la diversión con otros (Moreno-Murcia, González-Cutre \& Sicilia, 2007). Weiss y Chaumeton (1992) señalaron la relevancia que representa para los jóvenes el hecho de pertenecer a un grupo. Estudios relacionados con la afiliación y el reconocimiento social señalan la trascendencia de la afiliación en el sexo femenino por motivos de participación (Moreno-Murcia, Marcos-Pardo \& Huéscar, 2016) mientras que los trabajos centrados en los chicos apuntan a la pertenencia a un grupo (Kilpatrick, Hebert \& Bartholomew, 2005; Moreno-Murcia, Marcos-Pardo \& Huéscar, 2016).

Por todo lo expuesto, el presente estudio pretende analizar las preferencias de interacción social según edad y sexo, de los jugadores y jugadoras de las Escuelas Sociodeportivas de Baloncesto de la FRM.

\section{Método}

\section{Participantes}

La población objeto de estudio estuvo compuesta por la totalidad de jugadores pertenecientes a las Escuelas Sociodeportivas de Baloncesto de la FRM de la Comunidad, de Madrid, de las categorías benjamín (8-10 años), alevín (10-12 años), infantil (12-14 años) y cadete (14-16 años). De esta forma, la muestra fue de 413 sujetos (281 hombres y 132 mujeres), de los cuales 134 llevaban una temporada (nóveles) y 279 más de una temporada (veteranos) participando en las Escuelas Sociodeportivas.

\begin{tabular}{|c|c|c|c|c|c|}
\hline & Benjamín & Alevín & Infantil & Cadete & Total \\
\hline Hombre & $87(31 \%)$ & $93(33,1 \%)$ & $75(26,7 \%)$ & $26(9,3 \%)$ & 281 \\
\hline Mujer & $42(31,8 \%)$ & $56(42,4 \%)$ & $29(22 \%)$ & $5(3,8 \%)$ & 132 \\
\hline Total & $129(31,2 \%)$ & $149(36,1 \%)$ & $104(25,2 \%)$ & $31(7,5 \%)$ & 413 \\
\hline
\end{tabular}

\section{Instrumento}

Se utilizó la Escala Graupera/Ruiz de preferencias de interacción social en educación física (GR-SIPPEL) (Ruiz, Graupera \& Mata, 2004). El cuestionario está formado por 28 ítems divididos en cuatro dimensiones de siete ítems cada una:

- Cooperativa: se caracteriza por querer realizar los ejercicios mejor que los demás.

- Competitiva: caracterizada por el deseo de compartir, de trabajar en grupo.

- Afiliativa: se caracteriza por considerar la participación como un medio para ser integrado y aceptado por el grupo.

- Individualista: caracterizada porque el éxito se consigue sin ayuda de los demás.

Cada ítem se mide a través de una escala Likert de 4 opcio- nes donde, 1 significa muy en desacuerdo y, 4 totalmente de acuerdo. El análisis de fiabilidad del mismo, teniendo en cuenta la muestra del presente estudio, mostró valores adecuados respecto a la consistencia interna del instrumento, obteniendo un alfa de Cronbach total del cuestionario de ,726. Respecto al alfa por dimensión se obtuvieron los siguientes datos: cooperativa (,732), competitiva (,819), afiliativa (,786) e individualista (,644).

La escala GR-SIPPEL, creada en un principio para conocer las preferencias de interacción social de los alumnos en las clases de educación física, es susceptible a ser utilizada en contextos extraescolares. El deporte dentro de las clases de educación física, como aquél realizado fuera del ámbito educativo formal, deberían poseer características comunes en muchos sentidos, especialmente en relación con el desarrollo de valores educativos (Giménez \& Rodríguez, 2006). De forma similar, trabajos como el de Smith (2015) o Aoyagi et al. (2016) se centran en el estudio de aquellos aspectos que relacionan o complementan el deporte practicado en clase de educación física con el deporte extraescolar.

\section{Procedimiento}

La investigación se llevó a cabo una vez que la junta de revisión de ética de FRM aprobó el estudio. Además, los padres proporcionaron consentimiento informado por escrito. También se informó a los participantes sobre la finalidad del estudio y se garantizó que la información recogida seguiría siendo confidencial, como establece la Ley Orgánica 15/1999, de 13 de diciembre, de Protección de Datos de Naturaleza Personales.

Los cuestionarios se administraron al comienzo de la temporada 2014/15. Previamente a la aplicación del cuestionario se llevó a cabo una reunión informativa con los coordinadores de las escuelas Sociodeportivas, en donde se les explicó el objetivo del estudio, así como el protocolo de actuación. A continuación, los cuestionarios se pasaron en el lugar habitual de entrenamiento. Los coordinadores en presencia de los entrenadores de las respectivas Escuelas, realizaron una lectura detallada de cada ítem a fin de que todos los encuestados entendieran las preguntas, realizando las clarificaciones oportunas a los jugadores.

\section{Análisis estadístico}

Se llevó a cabo un análisis descriptivo básico de tendencia central y dispersión de cada una de las dimensiones. Se realizó la prueba de normalidad. Para ello se utilizó la prueba Kolmogorov-Smirnov, mostrando los resultados la necesidad de realizar pruebas no paramétricas $(p<, 05)$. Para determinar si existían diferencias significativas dependiendo del género, se usó la prueba de contraste U de Mann-Whitney. Para las comparaciones intergrupales, entre las distintas categorías, se utilizó la prueba de contraste Kruskal-Wallis. Además, en aquellos casos en los que las comparaciones intergrupo fueron significativas $(p<, 05)$, se realizó la comparación por pares a través del estadístico U de Mann-Whitney, aplicando el ajuste de Bonferroni para la corrección del nivel de significación, y se calculó el tamaño del efecto (Field, 2009).

Finalmente, para determinar si existían asociaciones entre las distintas dimensiones se utilizó el coeficiente de correlación de Spearman. El programa estadístico usado fue el SPSS en su versión 21.0.

\section{Resultados}

Las dimensiones más valoradas tanto en chicos como en chicas son por este orden: cooperativa, afiliativa, competitiva e individualista.

Al comparar cada dimensión en función del sexo, MannWhitney ofrece resultados estadísticamente significativos en relación con la competición ( $\mathrm{Z}=-2,536, p=, 011 ; r=-0,125)$, y 
a la afiliación $(Z=-2,562 ; p=, 010 ; r=-0,125)$, siendo los chicos más competitivos y más afiliativos que las chicas, aunque en ambos casos el tamaño del efecto es pequeño.

\section{Dimensión cooperativa}

La dimensión cooperativa no muestra diferencias significativas en relación con el sexo. Sin embargo, al analizarla en función de las distintas categorías se observó que disminuía conforme avanzaba la edad de los niños/as: benjamines $(\mathrm{M}=3,638 \pm$ ,421), alevines ( $M=3,547 \pm, 441)$, infantiles $(M=3,299 \pm, 441)$ y cadetes $(M=3,244 \pm, 461)$, detectándose diferencias significativas $\left(X^{2}=54,447, p<, 001\right)$. Respecto a la comparación por pares de categorías, la prueba de contraste U Mann Whitney con corrección de Bonferroni, mostró que había diferencias significativas entre las categorías benjamín e infantil $(Z=-6,388$, $p<, 001, r=-0,418)$, con un tamaño del efecto grande; y entre las categorías benjamín y cadete $(Z=-4,564, p<, 001, r=$ 0,361), alevín e infantil ( $Z=-4.907, p<, 001, r=-0,309)$, y alevín y cadete $(Z=-3,496, p<, 001, r=-0,261)$, cuyos tamaños del efecto muestran valores medianos.

\section{Dimensión afiliativa}

Los datos obtenidos en cada una de las categorías muestran que los valores, respecto a la media, van disminuyendo conforme se avanza en las distintas categorías, siendo más afiliativos los benjamines y menos los cadetes: benjamines $(\mathrm{M}=$ $3,132 \pm .548)$, alevines $(M=2,986 \pm, 505)$, infantiles $(M=2,737 \pm$ ,475) y cadetes $(M=2,682 \pm, 363)$, detectándose diferencias significativas $\left(X^{2}=39,418, p<, 001\right)$. Tras la comparación por pares, la prueba de contraste U Mann Whitney con corrección de Bonferroni, mostró que había diferencias significativas entre las categorías benjamín e infantil $(Z=-5,437 ; p<, 001 ; r=-0,356)$, benjamín y cadete $(Z=-4,374 ; p<, 001 ; r=-0,346)$, y entre alevín e infantil $(Z=-3,293 ; p<, 001 ; r=-0,207)$, y alevín y cadete $(Z=-3,086 ; p=, 002 ; r=-0,230)$. El tamaño del efecto muestra valores medianos en todos los casos.

La dimensión afiliativa mostró diferencias significativas teniendo en cuenta el sexo, siendo los chicos más afiliativos que las chicas. Atendiendo a las diferencias en función del sexo dentro de cada categoría, se observaron diferencias principalmente en las categorías benjamín ( $Z=-3,064 ; p=, 002 ; r=-0,270)$ y alevín $(Z=-3,217 ; p=, 001 ; r=-0,264)$, donde los chicos pre-

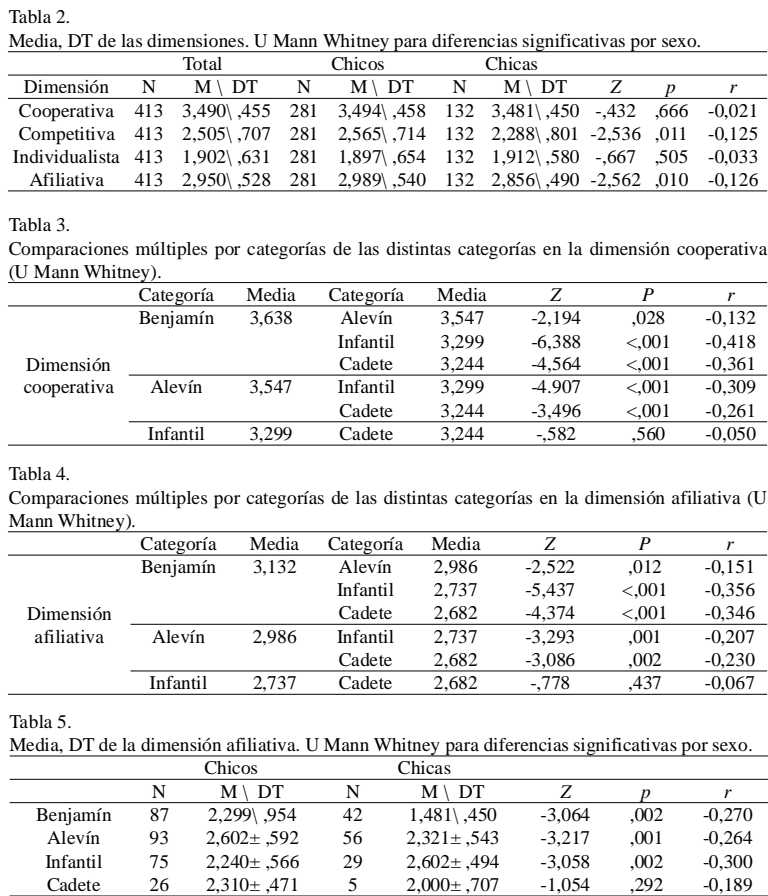

sentaron valores más elevados que las chicas; mientras que en la categoría infantil $(Z=-3,053 ; p=, 002 ; r=-0,299)$, fueron las chicas las que mostraron valores más altos.

Respecto al tiempo de práctica deportiva en la escuela, se observó que había diferencias significativas, $(Z=-2,346 ; p<, 019$; $r=-0,110)$, ya que los deportistas veteranos mostraron indicen más bajos respecto a las preferencias afiliativas: nóveles ( $\mathrm{M}=$ $2,620 \pm .585)$, veteranos $(M=2,470 \pm$,627).

\section{Dimensión competitiva}

En función de las distintas categorías se observó que la competición aumentaba conforme los chicos y chicas subían de categoría: benjamines $(M=2,351 \pm, 843)$, alevines $(M=2,540 \pm$ ,586), infantiles $(M=2,588 \pm, 536)$ y cadetes $(M=2,694 \pm, 536)$. Se detectaron, a priori, diferencias significativas $\left(X^{2}=11,105\right.$, $p=, 011)$.

Al realizar la comparación por pares de categorías, la prueba de contraste U Mann Whitney con corrección de Bonferroni, mostró que había diferencias significativas entre las categorías de minibasket (benjamín y alevín) y baloncesto (infantil y cadete), concretamente entre benjamín e infantil $(Z=-2,514 ; p=$ ,012; $r=-0,165)$. El tamaño del efecto mostró valores bajos.

La dimensión competitiva presentó diferencias significativas en relación con el sexo, siendo, en general, los chicos más competitivos. Atendiendo a las diferencias en función del sexo dentro de cada categoría, se observaron que las diferencias estaban, fundamentalmente, en las categorías alevín $(Z=-2,630$; $p=$ ,009; $r=-0,215)$, donde los chicos mostraron valores más elevados $(M=2,193 \pm, 595)$ que las chicas $(M=1,964 \pm, 660)$.

Respecto al tiempo de práctica deportiva en la escuela, se observó que había diferencias significativas, $(Z=-2,365 ; p<, 018$ $r=-0,116)$, ya que los deportistas veteranos mostraron indicen más altos respecto a las preferencias competitivas: nóveles ( $\mathrm{M}=$ $1,980 \pm .799)$, veteranos $(M=2,170 \pm, 723)$.

\section{Dimensión individualista}

La dimensión individualista no evidenció diferencias significativas en relación con el sexo. No se observó una evolución lineal de los valores obtenidos en un sentido creciente o decreciente a lo largo de las distintas categorías: benjamines ( $\mathrm{M}=$ $1,903 \pm, 680)$, alevines $(M=1,80 \pm, 595)$, infantiles $(M=2,027 \pm$ ,632) y cadetes $(M=1,959 \pm, 510)$. Se detectaron diferencias significativas $\left(X^{2}=1,497 ; p=, 015\right)$. Respecto a la comparación por pares de categorías, la prueba de contraste U Mann Whitney con corrección de Bonferroni, mostró que solamente había diferencias significativas entre las categorías alevín e infantil $(Z=-$ $3,153, p=, 002 ; r=-0,198)$, es decir, en el paso de las categorías de la modalidad de minibasket a baloncesto, aunque el tamaño del efecto en este caso es pequeño.

\section{Correlaciones entre las distintas dimensiones}

En relación con el grado de asociación entre las distintas dimensiones, se observó que había una relación lineal negativa y

Tabla 6

Comparaciones múltiples por categorías de las distintas categorías en la dimensión competitiva (U Mann Whitney).

\begin{tabular}{llllcccc} 
(U Mann Whitney). & & & & & & \\
\hline Categoría & Media & Categoría & Media & $Z$ & $P$ & $r$ \\
\hline \multirow{5}{*}{$\begin{array}{c}\text { Dimensión } \\
\text { competitiva }\end{array}$} & Benjamín & 2,351 & Alevín & 2,540 & $-2,194$ &, 016 & $-0,132$ \\
\cline { 2 - 8 } & & & Infantil & 2,588 & $-2,514$ &, 012 & $-0,165$ \\
& & & Cadete & 2,694 & $-2,339$ &, 019 & $-0,185$ \\
\cline { 2 - 8 } & Alevín & 2,540 & Infantil & 2,588 &,- 779 &, 436 & $-0,049$ \\
\cline { 2 - 8 } & & & Cadete & 2,694 & $-1,547$ &, 122 & $-0,115$ \\
\hline
\end{tabular}

Tabla 7

Comparaciones múltiples por categorías de las distintas categorías en la dimensión individualista (U Mann Whitney).

\begin{tabular}{cccccccc}
\hline & Categoría & Media & Categoría & Media & $Z$ & $P$ & $r$ \\
\hline \multirow{4}{*}{$\begin{array}{c}\text { Dimensión } \\
\text { Individualista }\end{array}$} & Benjamín & 1,903 & Alevín & 1,801 & $-1,161$ &, 246 & $-0,070$ \\
\cline { 2 - 8 } & & & Infantil & 2,027 & $-1,683$ &, 092 & $-0,110$ \\
& & & Cadete & 1,959 &,- 842 &, 400 & $-0,067$ \\
\cline { 2 - 8 } & & \multirow{2}{*}{1,801} & Infantil & 2,027 & $-3,153$ &, 002 & $-0,198$ \\
& & & Cadete & 1,959 & $-1,921$ &, 055 & $-0,143$ \\
\hline
\end{tabular}


estadísticamente significativa baja, entre las dimensiones cooperativa y competitiva $\left(r_{\mathrm{s}}=-, 112, p=, 022\right)$ y las dimensiones cooperativa e individualista $\left(r_{s}=-, 282, p<, 001\right)$. Por el contrario, se detectó una relación lineal positiva y estadísticamente significativa moderada, entre las dimensiones cooperativa y afiliativa $\left(r_{s}=, 476, p<, 001\right)$.

Por otro lado, se observó una relación positiva y estadísticamente significativa moderada, entre las dimensiones individualista y competitiva $\left(r_{s}=, 331,<, 001\right)$ y una relación lineal negativa y estadísticamente significativa baja, entre las dimensiones individualista y afiliativa $\left(r_{s}=-, 256, p<, 001\right)$.

Tabla 8.

Correlaciones por dimensiones (Rho de Spearman)

\begin{tabular}{|c|c|c|c|c|c|}
\hline & & Cooperativa & Competitiva & Individualista & Afiliativa \\
\hline Cooperativa & $\begin{array}{l}\text { Coefic. correlac. } \\
\text { Sig. (bilateral) }\end{array}$ & & & & \\
\hline Competitiva & $\begin{array}{l}\text { Coefic. correlac. } \\
\text { Sig. (bilateral) }\end{array}$ & $\begin{array}{c}-, 112 * \\
, 022\end{array}$ & & & \\
\hline Individualista & $\begin{array}{l}\text { Coefic. correlac. } \\
\text { Sig. (bilateral) }\end{array}$ & $\begin{array}{c}-, 288^{* *} \\
<, 001\end{array}$ & $\begin{array}{l}, 331^{* *} \\
<, 001\end{array}$ & & \\
\hline Afiliativa & $\begin{array}{l}\text { Coefic. correlac. } \\
\text { Sig. (bilateral) }\end{array}$ & $\begin{array}{l}, 476^{* *} \\
<, 001\end{array}$ & $\begin{array}{l}, 047 \\
, 338\end{array}$ & $\begin{array}{l}-, 256 * * \\
<, 001\end{array}$ & \\
\hline
\end{tabular}

\section{Discusión}

Las preferencias de interacción social en cualquier ámbito del deporte, ya sea en clase de educación física o en el seno de un club o escuela deportiva, van a estar marcadas por las características de los sujetos que lo conforman. En el contexto del aprendizaje deportivo se suelen dar relaciones intensas y afectivas entre los participantes y entre éstos y el entrenador, lo cual refleja que el aprendizaje es un fenómeno psicosocial complejo que implica tanto a unos como a otros y en el que va a influir el tipo de interacción social preferente de los mismos (Ruiz et al., 2004). En este sentido, se observa que los chicos y chicas pertenecientes a las escuelas sociodeportivas de Baloncesto de la FRM se inclinan más hacia el trabajo de forma cooperativa y afiliativa, mostrando valores superiores a los encontrados en otros estudios similares (Ruiz et al, 2004); mientras que, en menor medida, presentan preferencias hacia el aprendizaje con un carácter competitivo e individualista, lo cual coincide con lo encontrado por Rico (2003), Ruiz et al.(2004), y Graupera et al. (2010). Sin embargo, en relación con el sexo no se observan diferencias significativas en las dimensiones cooperativa e individualista, datos que no coinciden con los estudios de Farver y Branstetter (1994), Gabbei (2004) y Ruiz, et al. (2004), en el ámbito de la educación física, en los que los resultados mostraron que las chicas valoraban más que los chicos la dimensión cooperación. En este sentido, los planteamientos didácticos que caracterizan al modelo deportivo educativo de la FRM inciden en el desarrollo de valores como la igualdad, el compañerismo y el respeto, entre otros. Los distintos grupos de chicos y chicas de la FRM, en sus diferentes categorías, se caracterizan por la heterogeneidad. Por ello, se considera muy positivo el que las preferencias en cuanto al aprendizaje, en ambos sexos, se incline especialmente hacia el aprendizaje cooperativo.

Según diferentes autores, este tipo de aprendizaje tanto en el ámbito de la educación física como en el del deporte, facilita especialmente la inclusión del alumnado con diversidad funcional (André, Deneuve \& Louvet, 2011; Piercy, Wilton \& Townsend, 2002; Velázquez, Fraile \& López, 2014), favorece la incorporación de chicos y chicas de culturas diferentes (Oortwijn, Boekaerts \& VedderyFortuin, 2008), desarrolla la coeducación (Johnson \& Johnson, 2014), contribuye a la adquisición de las habilidades sociales y mejora las relaciones interpersonales (Dyson, 2001; Gillies \& Boyle, 2010; Goudas \& Magotsiou, 2009), y motiva a los chicos y chicas hacia la práctica motriz (Barba, 2010), aspectos sobre los que se hace hincapié en el proyecto deportivo de la FRM.
Según afirma Pujolàs (2012), pasar de una estructura de la actividad individualista y/o competitiva a una estructura de la actividad cooperativa es un elemento trascendental para hacer posible la inclusión de todo el alumnado. Por otra parte, los planteamientos cooperativos facilitan que los chicos y chicas de la FRM se esfuercen juntos y compartan recursos para mejorar su propio aprendizaje individual y colectivo, coincidiendo con lo expuesto por Velázquez $(2010,2015)$.

Por otro lado, se observa que las preferencias del aprendizaje cooperativo de los chicos y chicas van disminuyendo conforme avanzan en las distintas categorías, datos que se asemejan a los obtenidos en otros estudios realizados en el ámbito de la actividad física (Martínez, et al., 2008; Ruiz, et al., 2010), en los cuales se detectó que los niños conforme se hacen mayores son menos cooperativos. La justificación, según este último estudio, es que a medida que avanza la edad, los sujetos son más autónomos, por lo que consideran el espíritu y el trabajo de equipo un factor secundario.

La segunda dimensión más valorada es la dimensión afiliativa, lo cual coincide con lo hallado por Ruiz, et al., (2010). Otro dato en común entre dicho estudio y el presente trabajo es que las preferencias del aprendizaje relacionadas con la dimensión afiliativa van disminuyendo conforme avanza la edad de los encuestados. En este sentido, Martínez, Inglés, Piqueras y Oblitas (2010), y Silverio y García (2007), afirmaron que la etapa de la adolescencia se caracteriza por ser un periodo con perturbaciones temporales en diferentes áreas, y por ello los adolescentes se auto perciben y se consideran más inadaptados que los niños o los adultos. El motivo de afiliación hacia el grupo de iguales varía a lo largo del ciclo evolutivo, de modo que chicos y chicas de 13 años reconocen sentirse insatisfechos en la relación con sus iguales, mientras que los que tienen los 16 y 17 años comienzan a sentirse integrados en el grupo de iguales (Rodrigo et al., 2004).

Por otro lado, se detectan diferencias significativas respecto al sexo, siendo los chicos los que valoran más la afiliación, datos que coinciden con los estudios de Castillo y Balaguer (2001), y Kilpatrick, Hebert y Bartholomew (2005). Sin embargo, Ruiz, et al., (2010) hallaron datos opuestos, ya que las chicas presentaron mayor preferencia que los chicos en relación con la afiliación. Según Garita (2006), la afiliación, entre otros aspectos, es una variable importante para que niños y adolescentes hagan deporte. En cuanto al tiempo de práctica deportiva, se observa que aquellos que llevan más tiempo en las escuelas muestran menos preferencias afiliativas. Ruiz et al., (2004) señalan que la afiliación es el medio de ser acogido, reconocido e incluso querido en un grupo, por lo que es comprensible que esta actitud sea más propia de deportistas que entran a formar parte en un grupo social nuevo.

Respecto a la dimensión competitiva, se aprecian diferencias significativas en relación con el sexo, ya que los chicos muestran una mayor inclinación que las chicas hacia las preferencias relacionadas con aspectos competitivos, datos que se asemejan a los obtenidos por Ruiz et al. (2004), Kilpatrick, Hebert y Bartholomew (2005) y Ruiz, et al. (2010).

Los resultados obtenidos en el presente estudio contribuyen a afianzar los datos señalados en numerosas investigaciones relacionadas con el ámbito de la actividad física y el deporte, en las que se indica que el sexo femenino suele otorgar menos importancia a los aspectos relacionados con la competición (Hellín, Moreno \& Rodríguez, 2004; Kilpatrick et al., 2005; Moreno, Cervelló \& Martínez, 2007; Weinberg et al., 2000; Wilson \& Rodgers, 2002).

Al contrario de lo que ocurre con la dimensión cooperativa, los datos obtenidos en la dimensión competitiva van adquiriendo valores más elevados conforme aumenta la edad de los chicos y chicas, datos que no coinciden con los obtenidos por Ruiz et 
al. (2010), quienes encontraron que en la dimensión competitiva no se mostraba un aumento conforme avanzaba la edad de los sujetos. Hay que resaltar que dicho trabajo se encuadra en un ámbito de actuación de las clases de educación física, contexto diferente al de una escuela o club deportivo. El trabajo dentro de una escuela o club deportivo se caracteriza, entre otros aspectos, por trabajar en torno a una sola disciplina deportiva, lo que hace que los alumnos y alumnas a lo largo del tiempo se sientan más competentes en la disciplina deportiva, debido a la adquisición y asimilación de las habilidades táctico-técnicas de dicho deporte. Por ello, aunque en el presente trabajo sí se observa un aumento en cuanto a los valores medios obtenidos, no se puede afirmar que los datos presenten incrementos estadísticamente significativos, lo cual avala los planteamientos didácticos en los que se fundamenta las escuelas sociodeportivas de la FRM, en donde la competición se plantea y desarrolla bajo los principios de participación y autonomía, ya que los chicos y chicas diseñan la composición de los equipos, se responsabilizan de los cambios de jugadores en los torneos y se auto arbitran (Ortega el al., 2014). Además, se fomenta la igualdad, el respeto y el compañerismo, por las características heterogéneas de los sujetos que conforman los equipos, ya que éstos están formados tanto por niños como niñas, y en ocasiones, incluso, con chicos y chicas con diversidad funcional.

Otro aspecto a resaltar es el hecho de que la dimensión competición es una de las menos valoradas, sólo por encima de la dimensión individualista. En estudios relacionados con otras modalidades deportivas de equipo, como el fútbol, se observó que los factores relacionados con la competición están entre los aspectos más valorados por los deportistas de las diversas categorías, siendo las categorías alevín e infantil las que más la valoran (Martínez, et al., 2008). Guillén, Weis y Navarro (2005) señalan que el deporte durante la etapa de iniciación deportiva refleja matices rescatados de la sociedad actual, donde destacan fundamentalmente los resultados y la excelencia.

Para finalizar la dimensión competitiva, se ha de destacar que los deportistas con mayor tiempo de práctica muestran valores más elevados en la dimensión competitiva. Hutchins (1978) y Ruiz et al. (2004), y en sus trabajos sobre actitudes cooperativas y competitivas en edades escolares, encontraron que había sujetos con un alto grado de competitividad a la vez que se mostraban muy cooperativos.

La dimensión individualista es la menos valorada, lo cual coincide con los estudios realizados, entre otros, por Ruiz, et al. (2010), en el ámbito de la educación física. Esta dimensión no muestra diferencias significativas en relación con el sexo. Además, no se observa una evolución lineal de los valores obtenidos en sentido creciente o decreciente a lo largo de las distintas categorías deportivas. Estudios que relacionaron la autoestima y las destrezas deportivas de los chicos y chicas, señalaron que los sujetos con mayores niveles de habilidades deportivas tienden a ser más individualistas (García \& García, 2006). La autoestima, es uno de los valores sobre los que se sustenta el proyecto «Por una educación real: valores y deporte», por lo que podemos considerar óptimos los resultados obtenidos, ya que, a pesar de que los grupos de chicos y chicas se caracterizan por su heterogeneidad, no se detectan diferencias respecto a las preferencias individualistas, en cuanto al sexo y categorías. En esta línea, Mora, Sousa y Cruz (2014) señalan la necesidad y la importancia de estudiar el rol de padres y entrenadores en relación con la formación de la autoestima de los niños y niñas.

En cuanto a las correlaciones entre las distintas dimensiones, se aprecia que hay una correlación moderada entre las dimensiones cooperativa y afiliativa, datos que se asemejan a los obtenidos por Ruiz et al., (2004), Ruiz et al. (2010), aunque en dichos estudios la correlación detectada era muy baja. Por otra parte, se observa una correlación moderada entre las dimensio- nes individualista y competitiva, datos similares a los expuestos por García y García (2006), quienes detectaron que los niños y niñas que mostraban actitudes más competitivas además eran más individualistas.

\section{Conclusiones}

Las dimensiones más valoradas, tanto en chicos como en chicas, son la cooperativa y la afiliativa; siendo las menos valoradas las dimensiones competitiva e individualista. En cuanto al sexo, se detectan diferencias significativas en las dimensiones competitiva y afiliativa.

Respecto a la dimensión cooperativa y afiliativa se observa que ambas disminuyen conforme avanza la edad de los niños/as, encontrándose diferencias significativas en el paso de las categorías de minibasket a baloncesto. En la dimensión competitiva no se detectan diferencias significativas a lo largo de las distintas categorías.

En relación con la dimensión individualista, las diferencias nuevamente aparecen entre el paso de las categorías de minibasket a baloncesto.

$\mathrm{Al}$ analizar el grado de asociación entre las distintas dimensiones se observa que los sujetos más cooperativos tienden a ser más afiliativos, y menos competitivos e individualistas, mientras que los que se inclinan por ser más individualistas también suelen ser más competitivos. Además, los chicos y chicas presentan actitudes más cooperativas frente a las competitivas. Finalmente, al comparar los datos obtenidos con los encontrados en otros estudios similares, se observa que el programa de escuelas sociodeportivas de baloncesto de la FRM, puede contribuir a que no existan diferencias acentuadas en función al sexo.

Cabe resaltar que la principal implicación práctica de los resultados hallados en esta investigación hace referencia a la necesidad y a la conveniencia de planificar y programar adecuadamente la educación en valores a través de la práctica deportiva. En cuanto a las limitaciones del estudio llevado a cabo, cabe mencionar el hecho de que el instrumento de investigación utilizado fue diseñado para analizar las preferencias de interacción social en el ámbito de la educación física escolar, con lo que los resultados obtenidos deben tomarse con cierta cautela; aunque, como hemos podido comprobar, existen ciertas similitudes, lo cual es importante destacar. Futuros trabajos de investigación podrían centrarse en profundizar en el conocimiento de las preferencias de interacción social a través de entrevistas y estudios de casos en los distintos contextos de enseñanza deportiva, así como el análisis de la implementación de programas con una intención educativa deliberada como el desarrollado por la FRM.

\section{Referencias}

André, A., Deneuve, P., \& Louvet, B (2011). Cooperative Learning in Physical Education and Acceptance of Students with Learning Disabilities. Journal of Applied Sport Psychology, 23(4), 474-485. https://doi.org/1,1080/1041320,2011,580826

Aoyagi, K., Ishii, K., Shibata, A., Arai, H., Fukamachi, H., \& Oka, K. (2016). Cooperative coaching: Benefits to students in extracurricular school sports. Journal of Physical Education and Sport, 16(3), 806. DOI:10.7752/jpes.2016.03128

Barba, J. J. (2010). Diferencias entre el aprendizaje cooperativo y la asignación de tareas en la Escuela Rural. Comparación de dos estudios de caso en una unidad didáctica de acrosport en segundo ciclo de primaria. Retos: Nuevas Tendencias en Educación Física, Deportes y Recreación, 18, 14-18.

Castillo, I., \& Balaguer, I. (2001). Dimensiones de los motivos de práctica deportiva de los adolescentes valencianos escolarizados. Apunts. Educación Física y Deportes, 1(63), 22-29.

Contreras, O., De la Torre, E., \& Velázquez, R. (2001). Iniciación deportiva. Madrid. Síntesis. 
De Rose Jr., D., Korsakas, P., Carlstron, B. L. \& Ramos, R. (2000) Síntomas de estrés precompetitivo en jóvenes deportistas brasileños. Revista de Psicología del Deporte, 9(1), 143-157.

Dyson, B. (2001). Cooperative learning in an elementary Physical Education program. Journal of Teaching in Physical Education, 20, 264-281,

Dyson, B. (2001). Cooperative learning in an elementary Physical Education program. Journal of Teaching in Physical Education, 20(3), 264-281. https://doi.org/1,1123/jtpe,2,3,264

Field, A. (2009). Discovering statistics using SPSS ( $3^{\text {a }}$ ed.). Sage.

Gabbei, R. (2004). Achieving balance: secondary physical education gender grouping options. Journal of Physical Education, Recreation y Dance, 75(3), 33-40, https://doi.org/1,1080/07303084,2004,10609250

García, L., \& García, C. (2006). La autoestima y el aprendizaje de destrezas motoras deportivas en niños de 6 a 8 años. Revista Iberoamericana de Psicología del Ejercicio y el Deporte, 1(1), 41-68.

Gillies, R. M., \& Boyle, M. (2010). Teachers' reflections on cooperative learning: Issues of implementation. Teaching and Teacher Education, 26, 933-940. https://doi.org/1,1016/j.tate,2009,1,034

Giménez, F.J., \& Rodríguez, J.M. (2006). Buscando el deporte formativo ¿cómo formar a los maestros? Retos. Nuevas Tendencias en Educación Física, Deporte y Recreación, 9, 40-45.

Giménez, F.J., Abad, M.T., \& Robles, J. (2010). El proceso de formación del jugador durante la etapa de iniciación deportiva. Apunts. Educación Física y Deportes, 99, 47-55.

Goudas, M., \& Magotsiou, E. (2009) The Effects of a Cooperative Physical Education Program on Students' Social Skills. Journal of Applied Sport Psychology, 21(3), 356-364. https://doi.org/1,1080/ 10413200903026058

Granero-Gallegos, A., \& Baena-Extremera, A. (2014). Predicción de la motivación autodeterminada según las orientaciones de meta y el clima motivacional en Educación Física. Retos. Nuevas Tendencias en Educación Física, Deporte y Recreación, 25, 23-27.

Guillén, F., Weis, G., \& Navarro, M. (2005). Motivos de participación deportiva de niños atendiendo a sus edades. Apunts. Educación Física y Deportes, 80(2), 29-36.

Gutiérrez, M. (2003). Manual sobre valores en la educación física y el deporte. Barcelona. Paidós.

Harvey, S., Kirk, D., \& O’Donovan, T. M. (2014). Sport education as a pedagogical application for ethical development in physical education and youth sport. Sport, Education and Society, 19(1), 41-62. https:// dx.doi.org/10.1080/13573322.2011.624594

Hellín, P., Moreno, J. A., \& Rodríguez, P. L. (2004). Motivos de práctica físico-deportiva en la Región de Murcia. Cuadernos de Psicología del Deporte, 4(1-2), 101-116.

Johnson, D. W.,y Johnson, R. T. (2014). Cooperative Learning in 21st Century. Anales de Psicología, 30(3), 841-851. https://doi.org/1,6018/ analesps,3,3,201241

Kilpatrick, M., Hebert, E., \& Bartholomew, J. (2005). College students' motivation for physical activity: Differentiating men's and women's motives for sport participation and exercise. Journal of American College Health, 54, 84-94. https://doi.org/1,3200/JACH,54,2,87-94

Martínez, A. E., Inglés, C., Piqueras, J. A., \& Oblitas, L. A. (2010). Pape de la conducta prosocial y de las relaciones sociales en el bienestar psíquico y físico del adolescente. Avances en Psicología Latinoamericana, 28(1), 74-84

Martínez, R., Molinero, O., Jiménez, R., Salguero, A., Tuero, C., \& Márquez, S. (2008). La motivación para la práctica en la iniciación al fútbol: influencia de la edad/categoría competitiva, el tiempo de entrenamiento y la relación con el entrenador. Apunts. Educación Física y Deportes, 93(3), 46-54.

Monjas Aguado, R., Ponce Garzarán, A., \& Gea Fernández, J. (2015). La transmisión de valores a través del deporte. Deporte escolar y deporte federado: relaciones, puentes y posibles trasferencias. RETOS. Nuevas Tendencias en Educación Física, Deporte y Recreación, 28, 276-284

Mora, À., Sousa, C., \& Cruz, J. (2014). El clima motivacional, la autoestima y la ansiedad en jugadores jóvenes de un club de baloncesto. Apunts. Educación Física y Deportes, 117(3), 43-50. https://doi.org/1,5672/ apunts,2014-0983,es.(2014/3),117,04

Moreno, J. A., Cervelló, E., \& Martínez, A. (2007). Validación de la Escala de Medida de los Motivos para la Actividad Física-Revisada en españoles: Diferencias por motivos de participación. Anales de Psicología, 23(1), 167-176.

Moreno-Murcia, J. A., Pardo, M., Jorge, P., \& Huéscar, E. (2016). Motivos de práctica físico-deportiva en mujeres. Revista de Psicología del Deporte, 25(1), 35-41,
Moreno, J. A., González-Cutre, D., \& Sicilia, A. (2007). Metas sociales en las clases de educación física. Análisis y Modificación de Conducta, 33, 351-368.

Oortwijn, M. B., Boekaerts, M., Vedder, P. \& Fortuin, J. (2008). The impact of a cooperative learning experience on pupils' popularity, noncooperativeness and interethnic bias in multiethnic elementary schools. EducationalPsychology, 28(2), 211-221,

Ortega, G., Durán, L.J., Franco, J., Giménez, F.J., Jiménez, P.J., \& Jiménez, A.C. (2014). Compete to share. Madrid: Fundación Real Madrid.

Ortega, G., Franco, J., Giménez, F.J., Durán, L.J., Jiménez, A.C., Jiménez, P.J., \& Lambert, J. (2016). An evaluation of the «White Card» as a resource for promoting an educational sports competition. Journal of Human Sports and Exercise, 11(1), 19-30.

Ortega, G., Giménez, F.J., Franco, J., Durán, L.J., Jiménez, P.J., \& Jiménez, A.C. (2012). Iniciación al valorcesto. Madrid. Fundación Real Madrid.

Piercy, M., Wilton, K., \& Townsend, M. (2002). Promoting the social acceptance ofyoung children with moderate-severe intellectual disabilities using cooperative learning techniques. American journal on mental retardation, 107(5), 352-360. https://doi.org/1,1352/08958017(2002)107<0352:PTSAOY >2,0,CO;2

Prat, M., \& Soler, S. (2003). Actitudes, valores y normas en la educación física y el deporte. Barcelona. Inde.

Prieto, J. M. (2016). Relación entre competitividad, ansiedad social y compromiso con variables deportivas y académicas en futbolistas jóvenes. Revista Iberoamericana de Psicología del Ejercicio y el Deporte, 11(2), 193-200.

Pujolàs, P. (2012). Aulas inclusivas y aprendizaje cooperativo. Educatio siglo XXI: Revista de la Facultad de Educación, 30(1), 89-112,

Rico, I. (2003). Estructuras de interacción y clima motivacional en los escolares de la ESO en E.F. Tesis doctoral inédita. Universidad de Castilla la Mancha.

Rodrigo, M.J., Márquez, M.L., García, M., Mendoza, R., Rubio, A., Martínez, A., \& Martín, J.C. (2004). Relaciones padres-hijos y estilos de vida en la adolescencia. Psicothema, 16(2), 203-210,

Ruiz, L. M., Graupera, J. L., \& Mata, E. (2004). Preferencias participativas en educación física de los chicos y chicas de la educación secundaria mediante la «escala GR de participación social en el aprendizaje». Motricidad. European Journal of Human Movement, 12, 151-168.

Ruiz, L. M., Graupera, J. L., Moreno, J. A., \& Rico, I. (2010). Social Preferences for learning among adolescents in secondary Physical Education. Journal of Teaching in Physical Education, 29, 3-20. https://doi.org/1,1123/jtpe,29,1,3

Smith, A. (2015). Primary school physical education and sports coaches: evidence from a study of School Sport Partnerships in north-west England. Sport, Education and Society, 20(7), 872-888. http:// doi.org/ 10.1080/13573322.2013.847412

Twietmeyer, G. (2007) Suffering play: can the time spent on play and games be justified in a suffering world, Quest, 59, 201-211. h t t p : / / dx.doi.org/10.1080/00336297.2007.10483548

Velázquez, C. (2015). Aprendizaje cooperativo en Educación Física: estado de la cuestión y propuesta de intervención. Retos: Nuevas Tendencias en Educación Física, Deporte y Recreación, 28, 234-239.

Velázquez, C. (Coord.) (2010). Aprendizaje cooperativo en Educación Física. Fundamentos y aplicaciones prácticas. Barcelona: Inde.

Velázquez, C., Fraile, A., \& López, V. M. (2014). Aprendizaje cooperativo en Educación Física. Movimento: Revista da Escola de Educação Física, 20(1), 239-259.

Veroz, R., Yague, J.M., \& Tabernero, B. (2015). Incidencia de dos modelos de competición de fútbol sobre los valores socio-educativos en prebenjamines. Retos: Nuevas Tendencias en Educación Física, Deporte y Recreación, 28, 84-89.

Weinberg, R., Tenenbaum, G., McKenzie, A., Jackson, S., Anshel, M., Grove, R., \& Fogarty, G. (2000). Motivation for youth participation in sport and physical activity: relationships to culture, self-reported activity levels, and gender. International Journal of Sport Psychology, 31, 321-346.

Weiss, M. R., \& Chaumeton, N. (1992). Motivational orientations and sport behavior. En T. Horn (ed.), Advances in Sport Psychology (pp. 101-184). Champaign, IL: Human Kinetics.

Wilson, P. M., \& Rodgers, W. M. (2002). The relationship between exercise motives and physical self-esteem in female exercise participants: An application of self- determination theory. Journal of Applied Biobehavioral Research, 7, 30-43. https://doi.org/1,1111/j,17519861,2002,tb00074.x 